\section{Resultados de miringoplastia Inlay com cartilagem de tragus}

\section{Inlay tragus cartillage mirringoplasty results}

\author{
Couto L.G.F. ${ }^{1}$, Marquezini R.M.S. ${ }^{2}$, \\ Oliveira J.A.A. ${ }^{3}$, Massuda E.T.
}

Palavras-chave: miringoplastia,
cartilagem de tragus, enxerto.
Key words: tragus cartilage,
miringoplasty, graft.

\section{Resumo / Summary}

\begin{abstract}
T
he use of tragus cartilage to close tympanic membrane (TM) perforations with transcanal approach was first described by Eavey in 1998 with excellent results for graft "take-rate" and with advantages such as technique ease, rapidity and postoperative patient comfort. Aim: To evaluate the inlay tragus cartilage miringoplasty results in patients underwent this technique at Clinical Hospital of Medicine School of São Paulo University of Ribeirão Preto - Department of Otolaringology during the last three years. Study design: Clinical prospective. Material and Method: For this study we used 32 surgeries performed at Clinical Hospital of Ribeirão Preto - Department of Otolaringology from 2000 to 2003 in patients with chronic otitis media and tympanic membrane perforations size at the most $5 \mathrm{~mm}$ of diameter without counterindications to perform the transcanal approach. Results: Closure of tympanic membrane was achieved in 28 of 32 surgeries performed, with $87.5 \%$ of success. Discussion: This study maintained a high rate of success to close tympanic membrane perforations by the Eavey technique principles described in other studies, despite of some modifications from the original technique. Conclusion: The tragus cartilage inlay miringoplasty with cartilage resembling butterfly wings has a high rate of success to close tympanic membrane perforations with $5 \mathrm{~mm}$ diameter at the most in cases of chronic otitis media with healthy middle ear. It gives more patient comfort with less morbidity.
\end{abstract}

\footnotetext{
${ }^{1}$ Médico residente do $3^{\circ}$ ano do Serviço de Otorrinolaringologia do Hospital das Clínicas da Faculdade de Medicina de Ribeirão Preto da Universidade de São Paulo. ${ }_{2}^{2}$ Médica residente do $1^{\circ}$ ano do Serviço de Otorrinolaringologia do Hospital das Clínicas da Faculdade de Medicina de Ribeirão Preto da Universidade de São Paulo. ${ }^{3}$ Professor Titular da disciplina de Otorrinolaringologia pelo Departamento de Oftalmologia, Otorrinolaringologia e Cirurgia de Cabeça e Pescoço do Hospital das Clínicas da Faculdade de Medicina de Ribeirão Preto da Universidade de São Paulo.

${ }^{4}$ Mestre em Otorrinolaringologia pela Faculdade de Medicina de Ribeirão Preto da Universidade de São Paulo e Médico Assistente da disciplina de Otorrinolaringologia pelo Departamento de Oftalmologia, Otorrinolaringologia e Cirurgia de Cabeça e Pescoço do Hospital das Clínicas da Faculdade de Medicina de Ribeirão Preto da Universidade de São Paulo. Endereço para correspondência: A/C Dr. José Antônio A. Oliveira - Departamento de Oftalmologia, Otorrinolaringologia e Cirurgia de Cabeça e Pescoço da Faculdade de Medicina de Ribeirão Preto - USP - Av. Bandeirantes, 3900 Ribeirão Preto SP $14049-900$ Tel (0xx16) 602-2863 - E-mail: ramancio@fmrp.usp.br

Artigo recebido em 26 de abril de 2004. Artigo aceito em 20 de maio de 2004.
} 


\section{INTRODUÇ̃̃O}

Desde que a cirurgia de miringoplastia passou a ser realizada em 1952 por Zöllner ${ }^{1}$ e Wullstein ${ }^{2}$, vários tipos de material têm sido utilizados como enxertos para o fechamento de perfurações de membrana timpânica (MT) como veia ${ }^{3}$, fáscia ${ }^{4}$, gordura ${ }^{5}$ e pericôndrio ${ }^{6}$. Destacam-se, nesse grupo, o uso de fáscia de músculo temporal e pericôndrio com grande sucesso. Paralelamente, o uso de cartilagem nas cirurgias de orelha média tem sido relatado com indicações precisas e algumas vantagens sobre os demais materiais, especialmente em casos de otite atelectásica, otite colesteatomatosa e miringoplastia revisional, apesar da discussão a respeito dos resultados quanto a audição nesses casos devido às características peculiares do enxerto de cartilagem ${ }^{6}$.

Especificamente para o fechamento de perfurações de MT com o acesso transcanal e técnica inlay, o uso de cartilagem foi primeiramente descrito por Eavey em 1998 cujo método mostra excelentes resultados no fechamento de perfurações não marginais de tamanho pequeno ou médio e com vantagens que incluem facilidade técnica, rapidez e conforto pós-operatório para o paciente .

Apesar da grande aplicabilidade e do sucesso dos primeiros resultados publicados, a literatura carece de maior quantidade de casos e maior follow-up dos pacientes no intuito de analisar o comportamento do enxerto a longo prazo, a ocorrência de insucessos e possíveis razões e a padronização da indicação da técnica quanto à idade dos pacientes e tamanho da perfuração.

No Brasil, Lubianca-Neto et al. mostraram os primeiros resultados brasileiros em 1998 apresentando 100\% de pega do enxerto em seus casos e melhora auditiva em todos eles ${ }^{8}$. Posteriormente, com uma casuística de 20 casos apresentaram um sucesso de $90 \%$ na pega do enxerto e analisaram os resultados auditivos decorrentes das cirurgias?. Estudaram, ainda, de forma randomizada, a técnica inlay com cartilagem e a técnica convencional com fáscia de músculo temporal e confecção de retalho timpano-meatal, não apresentando diferenças estatisticamente importantes nos resultados a despeito das maiores vantagens no uso da cartilagem inlay, a saber: menores cuidados pós-operatórios, menor custo para o sistema de saúde, baixa morbidade e maior conforto ao paciente ${ }^{10}$.

O presente estudo mostra os resultados obtidos com a miringoplastia inlay com cartilagem de tragus seguindo os princípios da técnica de Eavey em pacientes operados no serviço de Otorrinolaringologia do Hospital das Clínicas da Faculdade de Medicina de Ribeirão Preto da Universidade de São Paulo nos últimos 3 anos.

\section{OBJETIVO}

Avaliar os resultados da miringoplastia "inlay" com cartilagem de tragus seguindo os princípios da técnica de
Eavey, enfatizando pega do enxerto e melhora auditiva, através de audiometria pré e pós-operatória, em pacientes operados no serviço de Otorrinolaringologia do Hospital das Clínicas da Faculdade de Medicina de Ribeirão Preto da Universidade de São Paulo.

\section{MATERIAL E MÉTODO}

\section{Pacientes}

Foram utilizados para esse estudo 32 cirurgias realizadas no serviço de Otorrinolaringologia do Hospital das Clínicas da Faculdade de Medicina de Ribeirão Preto da Universidade de São Paulo no período de dezembro de 2001 a fevereiro de 2003. Foram descartados todos os casos operados e que, por qualquer razão, não realizaram avaliação auditiva pós-operatória. Cinco pacientes tiveram as duas orelhas operadas e cada orelha foi considerada como procedimento individual. A idade dos pacientes variou de 11 a 53 anos. Um paciente apresentava disacusia neurossensorial profunda pré-operatória. Nove casos tinham miringoplastia prévia com técnica convencional underlay com utilização de fáscia de músculo temporal.

O tamanho da perfuração de MT variou de $2 \mathrm{~mm}$ a $5 \mathrm{~mm}$ de diâmetro, sendo consideradas perfurações até $3 \mathrm{~mm}$ como pequenas e de 3 a $5 \mathrm{~mm}$ como perfurações moderadas. Todos os pacientes tinham diagnóstico de otite média crônica simples e apresentavam boas condições de orelha média. Foi realizada avaliação audiométrica pré e pós operatória em todos os pacientes levando-se em conta a média do gap (diferença entre os limiares por via aérea e por via óssea) nas freqüências de $500 \mathrm{~Hz}, 1000 \mathrm{~Hz}$ e $2000 \mathrm{~Hz}$. Foi considerado como fator de inclusão o intervalo entre via aérea e óssea menor que 30 dB nível sensação de audição, importante para indicação de miringoplastia.

Foram utilizados os seguintes parâmetros para a utilização da técnica inlay: tamanho da perfuração (até $5 \mathrm{~mm}$ ), visualização de toda a perfuração, otite média crônica simples com condições saudáveis de orelha média e ausência de irregularidades e tortuosidades de conduto auditivo externo que impossibilitassem o acesso endoaural.

\section{Procedimento}

Todas as cirurgias foram realizadas sob anestesia geral com intubação orotraqueal.

Após posicionamento do paciente e visualização microscópica da membrana timpânica foi realizada limpeza cuidadosa do conduto auditivo externo e escarificação delicada dos bordos da perfuração. Posteriormente, o tamanho da perfuração foi medido com um gancho de ângulo reto de $2 \mathrm{~mm}$ de comprimento. Todas as cirurgias foram realizadas através do acesso transcanal.

O enxerto de cartilagem de tragus foi retirado após incisão no domus tragal de cerca de $1,0 \mathrm{~cm}$ de extensão e dissecção da cartilagem dos tecidos adjacentes, mantendo o 
pericôndrio bilateralmente. A remoção da cartilagem respeitou a manutenção do suporte conferido pelo domus à estrutura do tragus. A pele foi fechada com pontos simples de fio nylon 5-0.

Observando a medida previamente realizada do tamanho da perfuração da MT, o enxerto de cartilagem foi confeccionado em forma de disco com bordos cerca de $2 \mathrm{~mm}$ maior que a perfuração. Foi mantido pericôndrio apenas em um dos lados do enxerto. Realizou-se incisão medial em toda a circunferência do enxerto, de tal forma que o resultado final mostrava duas lâminas de cartilagem unidas pelo centro, sendo uma delas recoberta por pericôncrio.

A cavidade da orelha média foi preenchida por gelfoam até cerca de $2 \mathrm{~mm}$ da superfície da perfuração. Após isso, o enxerto foi posicionado sob visualização microscópica na perfuração com técnica semelhante à colocação de um tubo de ventilação. O resultado final era a lâmina de cartilagem sem pericôndrio posicionada lateralmente à perfuração e aquela recoberta por pericôndrio medialmente à perfuração. O pericôndrio funcionava como uma cobertura dos bordos do enxerto. Toda a circunferência da perfuração foi checada. Não foi colocado nenhum enxerto de pele sobre a cartilagem posicionada como descrito inicialmente por Eavey com a utilização de fragmento de pele de braço ${ }^{4}$.

O curativo pós-operatório preconizava proteção com algodão otológico no primeiro dia, não molhar a orelha no primeiro mês de cirurgia e antibioticoprofilaxia com amoxicilina por 7 dias. Em casos de secreção mucóide ou purulenta presente na primeira semana de pós-operatório, prescreviam-se gotas com antibiótico por um período de dez dias.

\section{RESULTADOS}

Foi observado fechamento completo das perfurações de MT em 28 cirurgias das 32 realizadas após 1 mês de cirurgia perfazendo $87,5 \%$ de sucesso num seguimento pósoperatório de 4 anos e 7 meses (Gráfico 1). Nos casos de pega total, todos os enxertos apresentavam-se intactos e secos na última consulta (Figura 1). Não foi observada nenhuma complicação pós-operatória significativa. Nenhum problema relacionado à incisão de pele do tragus foi notificada.

As cirurgias foram realizadas por residentes do $2^{\circ}$ ano e por médicos assistentes e docentes do serviço de Otorrinolaringologia do Hospital das Clínicas da Faculdade de Medicina de Ribeirão Preto da Universidade de São Paulo havendo padronização da técnica realizada mas não do cirurgião.

Nos casos em que houve sucesso cirúrgico (28 pacientes), nove tinham perfurações moderadas (3-5mm) e 19 apresentavam perfurações pequenas (até $3 \mathrm{~mm}$ ).

$\mathrm{Na}$ avaliação auditiva pré e pós-operatórias, nos casos de pega total do enxerto, observamos os se guintes resultados: nove pacientes não apresentavam gap pré-operatório e 1 apresentava disacusia profunda; todos sem alteração audiométrica após o procedimento. Dezoito pacientes apresentavam gap pré-operatório que variou de 5 a 30 dB. Na audiometria pós-operatória, apenas 3 não apresentaram melhora auditiva e nos demais ( 15 casos) a média de melhora foi de 14,4 dB (Gráfico 2).

Nos casos de insucesso cirúrgico ( 4 casos) apenas 1 apresentou melhora audiométrica $(10 \mathrm{~dB})$ no pós-operatório (Gráfico 3).

\section{DISCUSSÃO}

A utilização de cartilagem no campo das cirurgias otológicas já foi amplamente estudada e descrita principalmente nas abordagens de patologias avançadas de orelha média como casos revisionais, nos casos de retração severa de MT, atelectasia, colesteatoma ou perfuração no sítio da tuba auditiva. Os resultados audiológicos com a utilização desse enxerto também foram descritos e não mostraram diferenças significativas com relação à utilização de outro material como fáscia de músculo temporal. Isso faz do enxerto de cartilagem uma ótima opção para indicações precisas de abordagem de patologias de orelha média ${ }^{6.11}$.

$\mathrm{Na}$ abordagem de casos de otite média crônica simples sem patologia avançada de orelha média, Eavey descreveu sua técnica inlay com cartilagem de tragus em forma de asas de borboleta para o fechamento de perfurações de MT com sucesso de 100\% ${ }^{7}$. Os resultados brasileiros com o uso dessa técnica mostrados por Lubianca-Neto et al. atingiram índice de pega do enxerto de 100\% e 90\%, respectivamente, nos dois estudos que realizou ${ }^{8,9}$.

O presente estudo manteve o alto índice de sucesso no fechamento das perfurações de MT ( $87,5 \%$ dos casos). Apesar dos mesmos princípios, algumas modificações foram feitas em relação à técnica original a saber: no grupo de pacientes também foram incluídos adultos, a orelha média foi preenchida com gelfoam antes do posicionamento do enxerto, conferindo sustentação e estabilização adicional à cartilagem, o pericôndrio foi mantido apenas na lâmina medial do enxerto, o que diminuiu a espessura da cartilagem e facilitou seu posicionamento não comprometendo a viabilidade da cartilagem ${ }^{12}$ e não foi utilizado enxerto de pele sobre a cartilagem, diminuindo assim a morbidade e o tempo cirúrgico sem prejuízo dos resultados. As modificações acima buscaram aperfeiçoar e adaptar a técnica original de acordo com a experiência de nosso serviço mantendo a alta eficiência da cirurgia.

Apesar do curto período médio de seguimento pósoperatório observamos que a cartilagem torna-se parte integrante da membrana timpânica e, como é da característica desse material, mantém-se nutrida por difusão. Trata-se de um material avascular que pode resistir a ambientes hostis e pobres em irrigação por longos períodos ${ }^{13}$. 


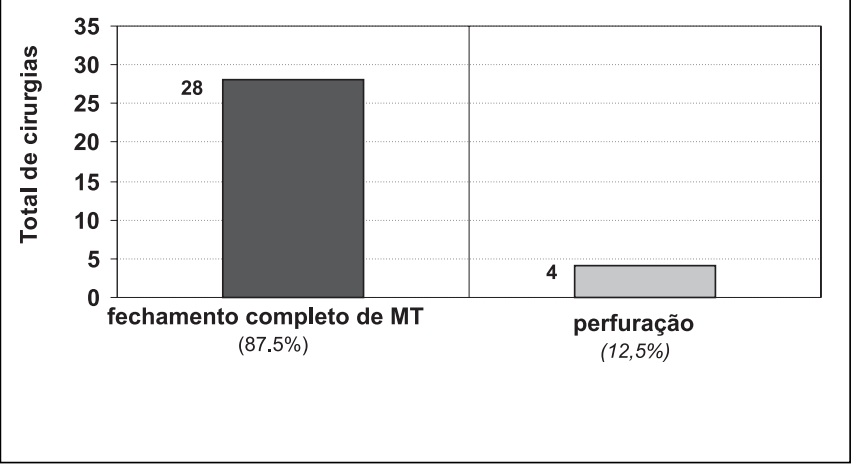

Gráfico 1. Resultados pós-operatórios

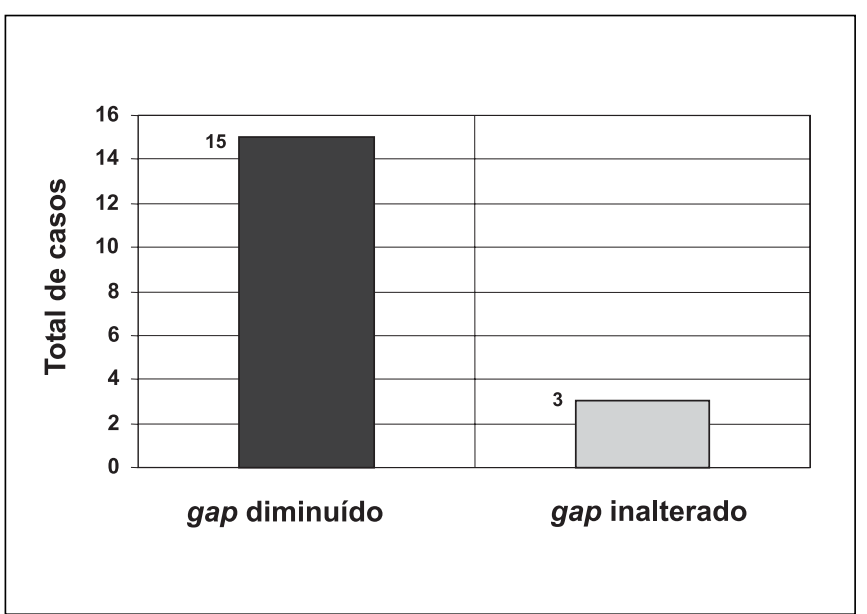

Gráfico 2. Avaliação auditiva dos casos com disacusia condutiva pré- operatória

O sucesso no fechamento das perfurações de MT também trouxe uma diminuição no gap pós-operatório em 83\% dos casos que tinham disacusia condutiva pré-operatória. Nos demais casos de sucesso cirúrgico, os limiares audiométricos não se modificaram. Vemos que não há qualquer influência que comprometa os resultados audiométricos com a utilização de cartilagem como enxerto de MT havendo até mesmo melhora auditiva na maioria dos casos de acordo com estudos previamente descritos ${ }^{7,8,9,10}$. Também acreditamos que quanto menos espesso for o enxerto confeccionado, menor será sua chance de comprometer ou de não melhorar a audição do paciente. É nesse sentido que removemos o pericôndrio de um dos lados do enxerto.

Ainda de acordo com a descrição original, também

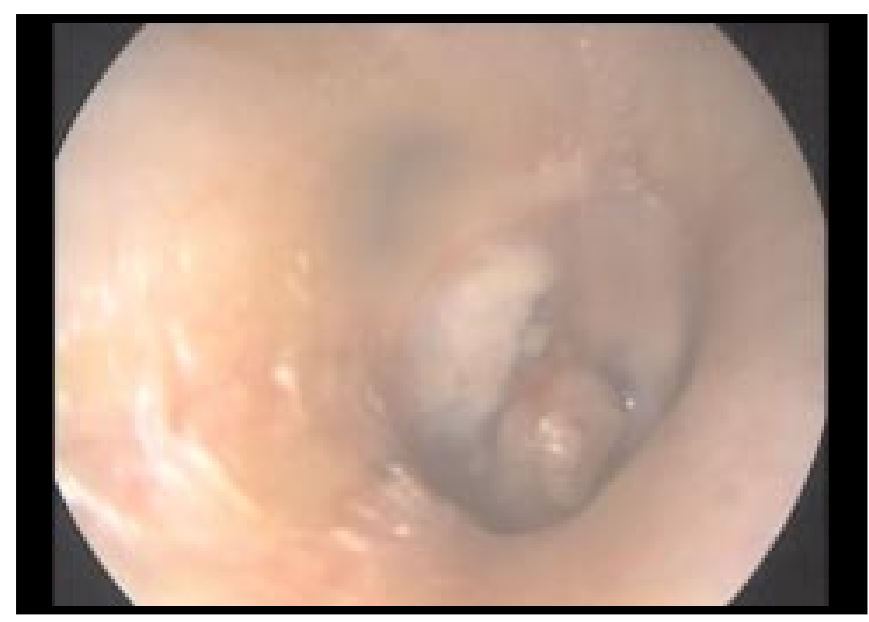

Figura 1. Aspecto da membrana timpânica após a cirurgia

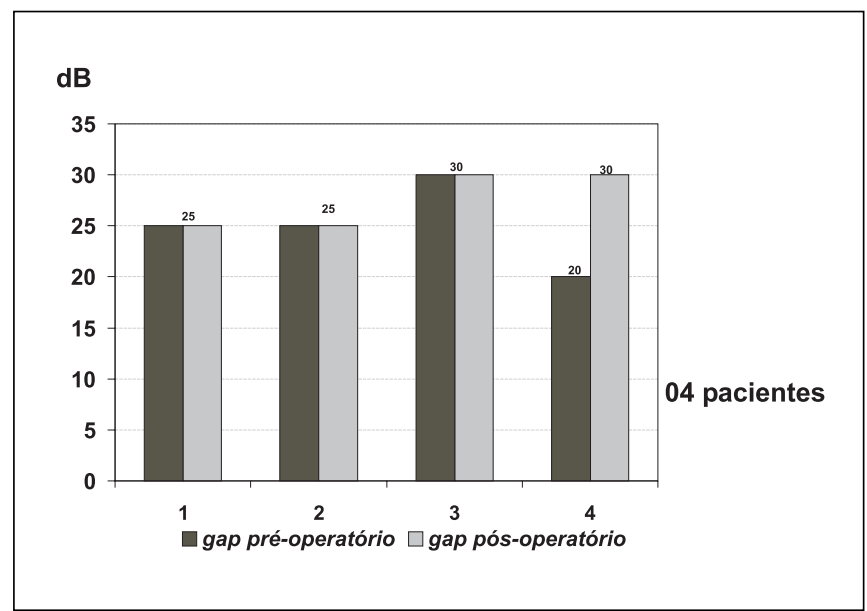

Gráfico 3. Avaliação auditiva dos casos com disacusia condutiva pré- operatória

observamos vantagens na utilização da técnica inlay com cartilagem de tragus devido à maior rapidez do procedimento, à não necessidade de confecção de retalho meatal ou de qualquer incisão no conduto auditivo externo, à não necessidade de qualquer curativo ou suporte específico no conduto auditivo externo e à facilidade de obtenção do enxerto. Tudo isso traduzido num maior conforto para o paciente com resultados altamente satisfatórios e semelhantes à técnica convencional com utilização de fáscia de músculo temporal ${ }^{7,10}$.

Respeitadas as indicações para a utilização da técnica em questão, vemos que a mesma não está indicada para os casos que requerem exploração da orelha média como desproporção entre a perda auditiva condutiva e o tamanho da 
perfuração, possível colesteatoma ou otorréia, perfurações marginais sem bordos visíveis, perfurações que não podem ser vistas totalmente via transcanal e membrana timpânica com processo granulomatoso extenso ${ }^{7}$.

Apesar das cirurgias do estudo terem sido realizadas por diferentes cirurgiões com experiência e capacidade técnica variáveis, o procedimento mostrou-se tecnicamente simples de ser realizado e cuja estabilidade do enxerto após seu correto posicionamento foi fator decisivo para o sucesso de $87,5 \%$ dos casos, observada a correta preparação do paciente para a cirurgia. Dessa forma, acreditamos que os quatro casos de insucesso desse estudo têm relação com o não ancoramento do enxerto em toda a sua circunferência nos bordos da perfuração e com infecção de ferida cirúrgica no pós-operatório imediato não controlada satisfatoriamente com antibioticoterapia tópica.

\section{CONCLUSÕES}

Este estudo mostrou que a miringoplastia inlay com cartilagem de tragus em forma de asas de borboleta tem alto índice de sucesso no fechamento das perfurações de membrana timpânica de até $5 \mathrm{~mm}$ de diâmetro em casos de otite média crônica simples onde pode-se visualizar todos os bordos da perfuração, onde não há patologias de orelha média e onde não é necessária a exploração da orelha média ou procedimentos em cadeia ossicular.

Trata-se de um procedimento que confere melhora auditiva na maioria dos pacientes que, na avaliação pré-operatória, apresentam perda auditiva condutiva.

Devido à facilidade técnica, rapidez na sua realização, acesso transcanal sem incisões e à fácil obtenção e confecção do enxerto, a cirurgia de Eavey e suas variantes até hoje descritas são uma ótima opção para miringoplastia nos casos de otite média crônica simples com características anteriormente relatadas conferindo maior conforto e menor morbidade ao paciente.

\section{REFERÊNCIAS BIBLIOGRÁFICAS}

1. Zöllner F. The principles of plastic surgery of the sound-conducting apparatus. J Laryngol Otol 1995; 69: 657-9.

2. Wullstein HL. Funktionelle Operationen im Mittelohr mit Hilfe des Freien Spaltlappentransplantates. Arch Otorhinolaryngol 1952; 161: 422-35.

3. Tabb HG. Closure of perforations of the tympanic membrane by vein grafts: a preliminary report of 20 cases. Laryngoscope 1960; 70: 271-4.

4. Herman H. Tympanic membrane plastic repair with temporalis fascia. Hals Nas Ohrennh 1960; 9: 136-9.

5. Ayache S, Braccini F, Facon F, Thomassin JM. Adipose graft: an original option in myringoplasty. Otol Neurotol 2003 Mar; 24(2): 158-64.

6. Dornhoffer JL. Hearing Results With Cartilage Tympanoplasty. Laryngoscope 1997; 107(8): 1094-9.

7. Eavey RD. Inlay Tympanoplasty: cartilage butterfly technique. Laryngoscope 1998 May; 108(5): 657-61.

8. Lubianca Neto JF. Miringoplastia com Cartilagem Inlay: Primeiros Resultados Brasileiros. Revista Brasileira de Otorrinolaringologia 2000; 66 (4): 310-4.

9. Lubianca-Neto JF. Inlay butterfly cartilage tympanoplasty (Eavey technique) modified for adults. Otolaryngol Head and Neck Surg 2000 Oct; 123(4): 492-4.

10. Mauri M. Evaluation of inlay buterfly cartilage tympanoplasty: a randomized clinical trial. Laryngoscope 2001; 111(8): 1479-85.

11. Gerber MJ, Mason JC, Lambert PR. Hearing results after primary cartilage tympanoplasty. Laryngoscope 2000 Dec; 110(12): 1994-9.

12. Smyth GDL, Kerr AG. Cartilage homografts. Experimental and clinical aspects. Acta Oto-rhino-laryngologica belg, 1970; 24: 53-9.

13. Levinson RM. Cartilage-perichondral composite graft tympanoplasty in the treatment of posterior marginal and attic retraction pockets. Laryngoscope 1987; 97: 1069-74. 